\title{
Dynamics of solar active regions
}

\section{Oscillations observed with MDI and their relation to the magnetic field topology}

\author{
K. Muglach ${ }^{1,2}$, A. Hofmann ${ }^{1}$, and J. Staude ${ }^{1}$ \\ 1 Astrophysikalisches Institut Potsdam, Telegrafenberg, 14473 Potsdam, Germany \\ e-mail: muglach@nrl.navy.mil \\ 2 Artep, Inc., Naval Research Laboratory, 4555 Overlook Ave. SW, Washington, DC 20375, USA \\ Received 30 June 2003 / Accepted 1 March 2005
}

\begin{abstract}
We present new results of an international joint observing campaign, which was carried out in September 2000 to study the oscillatory behaviour of solar active regions. We concentrate on oscillations in the lower layers of the solar atmosphere as observed with the Michelson Doppler Imager (MDI, Scherrer et al. 1995, Sol. Phys., 162, 129). The resulting photospheric oscillation power maps are compared with chromospheric maps from simultaneous UV continuum data (taken with the TRACE instrument). From a magnetic field extrapolation of the MDI magnetograms we infer that the reduction in chromospheric high frequency power and enhancement of photospheric high frequency power may be explained by the interaction of acoustic waves with the magnetic canopy.
\end{abstract}

Key words. Sun: photosphere - Sun: oscillations - Sun: magnetic fields

\section{Introduction}

The dynamic behaviour of solar active regions (ARs) has been the topic of the international joint observing campaigns, that were taking place in September 1999 and $2000^{1}$. By combining several instruments (at different observatories) and applying various observing techniques we could obtain data that cover all layers of the solar atmosphere. A special interest of these campaigns is the interaction of solar oscillations and the surface magnetic field. A variety of problems have been addressed in previous papers resulting from these campaigns: sunspot oscillations in umbrae and pores (Balthasar et al. 2000a,b; Muglach \& O'Shea 2001; O'Shea et al. 2002, 2003), intensity fluctuations propagating around spots (Georgakilas et al. 2002) and chromospheric oscillations of active regions in the UV (Muglach 2002, 2003).

In this contribution we will concentrate on photospheric oscillations as observed with the Michelson Doppler Imager (MDI, Scherrer et al. 1995) and compare them with oscillations found in UV images of the Transition Region and Coronal Explorer (TRACE, Handy et al. 1999). We investigate the spatial distribution of oscillatory power in an extended active region that contains a sunspot, surrounding plage and patches of quiet sun with network and internetwork.

\footnotetext{
1 JOP097, see

http://sohowww . nascom. nasa.gov/soc/J0Ps/jop097.txt
}

Previous large-scale 2D studies of photospheric AR dynamics were carried out by Brown et al. (1992), Hindman \& Brown (1998), Thomas \& Stanchfield (2000), Donea et al. (2000), Jain \& Haber (2002) and Finsterle et al. (2004). They found an increase of high frequency power $(v>5 \mathrm{mHz})$ in the surroundings of ARs in Doppler velocity power maps, also called power halos. These halos were not present in Doppler power maps at lower frequencies $(3 \mathrm{mHz})$. There is also a lack of power halos in continuum intensity power maps (Hindman \& Brown 1998; Jain \& Haber 2002). Jain \& Haber (2002) found that halos are also present in high frequency power maps of spectral line depth as observed with MDI. But this could also be due to a contamination from the Doppler shifts of the spectral line, as mentioned by the authors, similar to the Doppler-induced $B$ fluctuations found by Settele et al. (2002a). The actual locations of strong magnetic fields (sunspots, plage) have less oscillation power at all frequencies, which has been known for a long time. Chromospheric studies using Ca II K filtergrams also show high frequency power enhancements (Braun et al. 1992; Toner \& LaBonte 1993; Thomas \& Stanchfield 2000). On the other hand, when using TRACE UV continuum data Muglach (2003, Paper I) has shown that the areas around sunspots, plage and network regions have less high frequency power. Judge et al. (2001) also find such a decrease in power around the quiet sun network in their SUMER data and Krijger et al. (2001) find the same in their quiet sun UV data obtained with TRACE. 
The purpose of this follow-up paper is to provide a direct one-to-one comparison of photospheric and chromospheric power maps by using some of the TRACE results of Paper I and data obtained with the MDI on-board the SOHO spacecraft. This direct comparison is possible as both instruments observed the same active region (AR 9172) and the data is taken simultaneously. Unfortunately, only a single coordinated data set with the required data coverage and quality is available from JOP097. By using spacecraft data we can avoid spurious power signals that might be produced by seeing in groundbased data (Hill et al. 2001; Settele et al. 2002b). We present power maps in the two atmospheric layers, which leads to a new interpretation of the photospheric power halos, different from the ones suggested by previous investigations.

This paper is structured in the following way: Sect. 2 describes the MDI data and Sect. 3 shows the resulting power maps of MDI. Section 4 compares the MDI power maps with the power maps of TRACE and discusses their relation to the magnetic field structure and Sect. 5 summarizes the paper.

\section{Observations}

The MDI data was taken on September 29, 2000 from 09:18 UT to 11:52 UT. The TRACE sequence started at 08:06 UT but lasted for $4 \mathrm{~h}$ (see Paper I for details of the TRACE data). MDI was operating in its high-resolution mode which nominally gives a spatial resolution of about $1.2^{\prime \prime}$. The actual resolution is distorted towards the edge of the high-resolution field of view (HR FOV) to about twice of that value (see http://soi.stanford.edu/data/cal/all_data.html\# PlateScale). Intensity $I$, velocity $v$ and magnetic flux density $B$ were recorded at a cadence of $1 \mathrm{~min}$ (which gives a Nyquist frequency of $v_{\mathrm{Ny}}=8.33 \mathrm{mHz}$ ). The complete field of view covered an area of about $600^{\prime \prime} \times 300^{\prime \prime}$ (using the top part of the HR FOV). The data was downloaded from the MDI Web-archive, which provides data reduction of level 1.5. No additional data reduction was carried out. Using the location of the sunspot of the AR the images were derotated using the solar rotation law of Howard et al. (1990). Missing images were interpolated linearly. Finally, a temporal Fast Fourier Transform was calculated for each pixel in the maps. To display the results the power is integrated over two frequency ranges: $2.3-4.3 \mathrm{mHz}$ (the typical $5 \mathrm{~min} p$-mode frequencies) and $5.5-7.5 \mathrm{mHz}$ (high frequency oscillations with $2-3 \mathrm{~min}$ periods). MDI observes a Ni I line ( $\lambda 6768 \AA$ ) which is formed at a height of $h=100-200 \mathrm{~km}$ in the photosphere (Bruls 1993).

TRACE observations and data reduction are discussed in detail in Paper I. Here we use a $4 \mathrm{~h}$ time sequence of UV images at $1700 \AA$ covering the same time as MDI. The $1700 \AA$ bandpass contains primarily the UV continuum that is formed at the base of the chromosphere $(h=400-500 \mathrm{~km}$ in the quiet sun, Vernazza et al. 1981).

\section{Results}

Figure 1 shows the temporal average of the MDI magnetograms. The active region in the left part of the figure

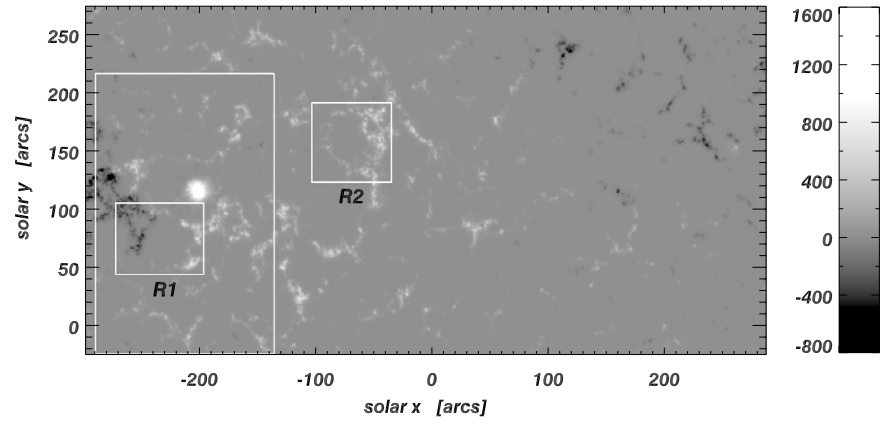

Fig. 1. Temporal average image of MDI magnetograms; the two magnetic polarities are given in black and white. The large white rectangle shows the FOV that was covered by TRACE (cf. Fig. 5). The two small rectangles indicate the regions $\mathrm{R} 1$ and $\mathrm{R} 2$ that were used for magnetic field extrapolations (shown in Fig. 4). The colorbar gives magnetic flux values.

(AR 9172) contains a bipolar region with a moderate-sized regular sunspot and surrounding plage area. Network and quiet sun internetwork can be seen further away from the AR. The two magnetic polarities are given in black and white. The large white rectangle outlines the TRACE FOV, the two smaller ones cover the parts that were used for a magnetic field extrapolation (see below). The magnetic field structure providing the topological context of the observed FOV is described in more detail in Paper I.

Figure 2 shows maps of oscillation power in MDI intensity $I$, the integrated 5 min power on top and the integrated 2-3 min power below. The TRACE FOV is again given by the large white rectangle. Each image is linearly scaled, the brighter the region, the higher the power. Figure 3 shows maps of oscillation power in MDI Doppler velocity $v$, again with 5 min power on top and 2-3 min power below. Comparing these figures with the ones published by Hindman \& Brown (1998), Donea et al. (2000) and Jain \& Haber (2002) (all of whom were using MDI data as well), we fully confirm their findings. The locations of high magnetic flux density (spot, plage and network) have reduced power at both frequency ranges, in both $I$ and $v$. The high frequency Doppler velocity map shows the power halos around strong magnetic fields, most pronounced in the AR in the left part of Fig. 3.

\section{Discussion}

In general a discussion of the wave modes in a stratified, compressible atmosphere permeated by a magnetic field $\boldsymbol{B}$ of arbitrary direction requires the simultaneous consideration of the three restoring forces due to magnetic pressure and tension, gas pressure $p_{\text {gas }}$ and buoyancy. The resulting MAG (magnetoacoustic-gravity) waves form a complicated system of oscillations which so far has been studied for simplifying special cases only. A first analytic approach has been published by Zhugzhda \& Dzhalilov (1984a,b). Recent numerical simulations in a two-dimensional geometry by Rosenthal et al. (2002) and Bogdan et al. (2003) demonstrate a bewildering complexity of wave patterns even in a rather simple magnetic structure. A direct numerical comparison of these theoretical results with 

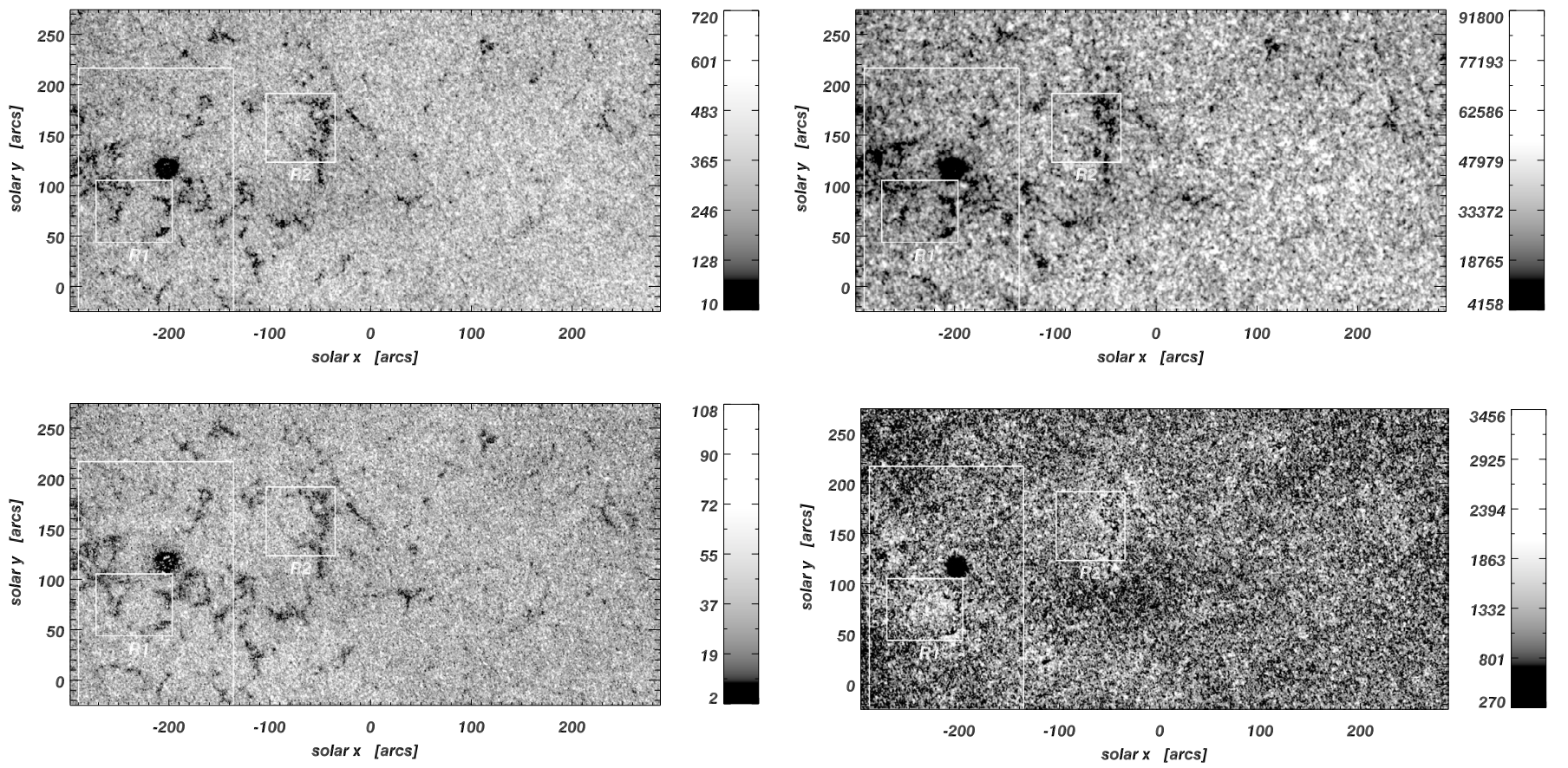

Fig. 2. Spatial distribution of power for MDI intensity $I$. The top image shows the integrated power between $2.3-4.3 \mathrm{mHz}$ ( 5 min range) and the bottom one between $5.5-7.5 \mathrm{mHz}(2-3$ min range). Dark areas have less power than bright ones. The large white rectangle shows the FOV that was covered by TRACE (cf. Fig. 5). The two small rectangles indicate the regions R1 and R2 that were used for magnetic field extrapolations (shown in Fig. 4). The colorbar gives arbitrary numbers.

observations is difficult due to the simplifying and restrictive assumptions present in these rather general approaches:

1. Two-dimensional structuring of the atmosphere and of $\boldsymbol{B}$ : any variation perpendicular to the plane containing the directions of gravity and $\boldsymbol{B}$ is neglected. In this case the pure Alfvén waves are completely decoupled from the other waves (the fast- and slow-mode MAG modes) and are no longer considered.

2. Isothermal atmosphere: in this case effects of a resonant transmission of MAG waves due to a temperature stratification are excluded. Such effects have been studied for sunspot umbrae assuming a vertical $\boldsymbol{B}$ by Žugžda et al. (1983, 1987), Gurman \& Leibacher (1984) and Settele et al. (1999, 2001).

For very strong $(\beta \ll 1)$ or very weak $\boldsymbol{B}(\beta \gg 1$, where the plasma $\beta=8 \pi p_{\text {gas }} / B^{2}$ ) the slow mode and the fast mode are decoupled from each other. Mode transformations (conversions) or reflections occur at the interaction region where the ratio of the thermal pressure $p_{\text {gas }}$ to the magnetic pressure is $\beta \approx 1$. This is the scenario that we used in Paper I to explain the reduced high frequency power in the TRACE maps shown in that paper: around the strong magnetic fields (at the photospheric level) we primarily find acoustic waves, the global $p$-modes. While waves below the acoustic cut-off frequency $v=v_{\mathrm{ac}} \approx 5 \mathrm{mHz}$ are reflected back into the lower photosphere, waves with $v>$ $5 \mathrm{mHz}$ can propagate upward into higher atmospheric layers. The inclined magnetic fields of the AR (spot, pore or cluster of

Fig. 3. Spatial distribution of power for MDI Doppler velocity $v$. The top image shows the integrated power between $2.3-4.3 \mathrm{mHz}$ ( $5 \mathrm{~min}$ range) and the bottom one between $5.5-7.5 \mathrm{mHz}$ (2-3 min range). Dark areas have less power than bright ones. The large white rectangle shows the FOV that was covered by TRACE (cf. Fig. 5). The two small rectangles indicate the regions $\mathrm{R} 1$ and $\mathrm{R} 2$ that were used for magnetic field extrapolations (shown in Fig. 4). The colorbar gives arbitrary numbers.

plage and network fluxtubes) produce the corrugated layers of mode conversion and reflection at $\beta \approx 1$, which is also called the "magnetic canopy".

The details of this wave interaction will eventually depend on the actual magnetic field distribution, especially the field topology in the higher layers. To get a better idea of this topology in our observed region we carry out potential field extrapolations by using the flux densities of MDI. We selected several regions for this magnetic field extrapolation as outlined in Fig. 1: the first one, R1, covers a large network cell near the sunspot and has both magnetic polarities. The second one, R2, is away from the AR and contains a unipolar network cell of approximately the same size as R1. We calculate the magnetic field following the procedure described by Seehafer (1978). Figure 4 shows the resulting 3D field distribution, R1 on the left and R2 on the right. The field lines reaching a height of $h=400-1000 \mathrm{~km}$ are drawn. In R1 they connect the opposite polarity fields. In the uni-polar region $\mathrm{R} 2$ field lines originating at the cell boundaries and reaching up to $5000 \mathrm{~km}$ are also included. The resulting field line distribution will always somewhat depend on the selection of the region on the solar surface. To check this we carried out several additional extrapolations of R1, selecting a larger FOV that also included the sunspot and the plage north of the network cell. The resulting field topology of R1 was still always the same. Nevertheless, the very distinct difference between the closed (R1) and the open field structure (R2) of the two cells is clearly visible. The bipolar network 

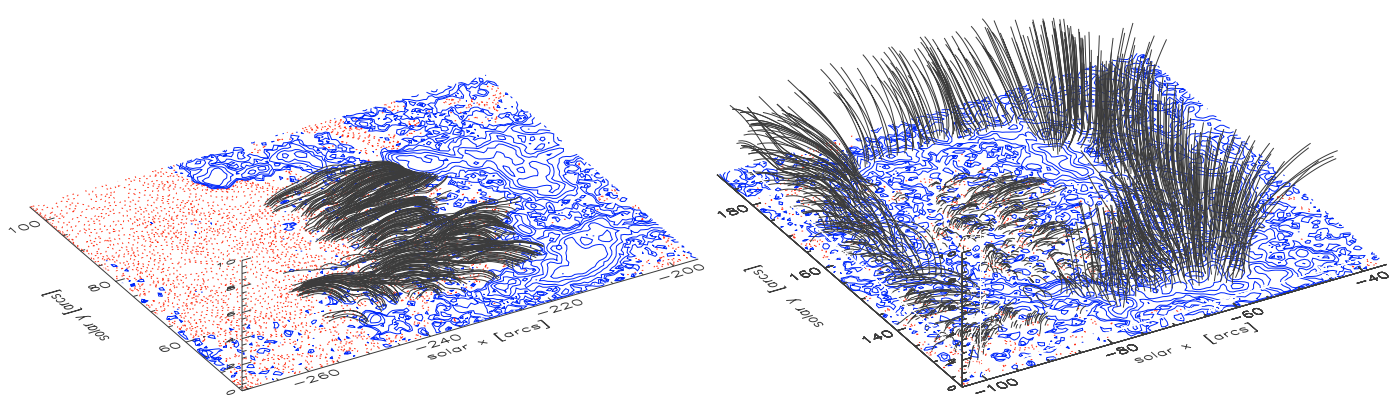

Fig. 4. Magnetic field extrapolation of two network cells: R1 (left) centered at $x=-230^{\prime \prime}, y=70^{\prime \prime}$ (in Fig. 1) which is also covered by the TRACE FOV and R2 (right) at $x=-70^{\prime \prime}, y=160^{\prime \prime}$ further away from the AR. The $x-y$ surface shows contours of equal magnetic field strength in solid and dashed lines for the two polarities. Fieldlines between $h=400-1000 \mathrm{~km}$ are included. The closed and open field configuration can be clearly seen.

cell is covered with a low-lying canopy of almost horizontal field lines. In the unipolar cell most of the field lines are only slightly inclined and reach up into the corona. When comparing the photospheric high frequency velocity power distribution of the two cells (Fig. 3) we can see that R1 shows a clear increase of power all across the cell. In R2 the increase is present only in the immediate surroundings of the network but not inside the cell.

In this paper we propose to explain the photospheric power halos as a signature of wave reflection of acoustic waves by the overlying inclined magnetic field. How much of the reflected waves we can actually see will depend on how "efficient" the reflecting layer is (how much transmission, reflection or wave conversion takes place) and of course on the angle between the incoming wave and the magnetic field. Part of the waves will simply be scattered away if the field is only slightly inclined to the direction of the wave. For an almost horizontal field, waves will primarily be reflected straight back down. In R1 with the low canopy overlying the complete cell this happens all across the cell, while in R2 it happens only near the border of the network. The average integrated high frequency power in the halos is about $70 \%$ higher compared to the average power in quiet regions. Hindman \& Brown (1998) also give an enhancement of power with frequency which peaks at $6 \mathrm{mHz}$ at about $60 \%$ (see their Fig. 3). If we attribute this to wave reflection at the magnetic canopy then about $70 \%$ of the waves return to the photosphere.

We also carried out a magnetic field extrapolation of the region that covers the TRACE FOV. From this we calculate the plasma $\beta$ for the complete TRACE FOV up to a height of $2400 \mathrm{~km}$. The gas pressure $p_{\text {gas }}$ is taken from the VAL 3C model atmosphere (Vernazza et al. 1981). The wavemagnetic field interaction region is in the range of $\beta \approx 1$ and we choose $\beta=0.5,1$ and 10. For the formation layer of the TRACE $1700 \AA$ A filter intensity we use $h=500 \mathrm{~km}$ (Fossum \& Carlsson 2004; Uitenbroek 2004).

Figure 5 shows the MDI $v$ power maps (top) and the $1700 \AA$ TRACE power maps from Paper I (bottom). The white contours give the location of $\beta=0.5$ (dotted line), $\beta=1$ (dashed line) and $\beta=10$ (solid line) where $h=500 \mathrm{~km}$. In this figure we can now directly compare the power maps of MDI and TRACE. Region R1 is outlined by the white rectangle in all four power maps. In the higher layers covered by the TRACE filter we find a strong decrease in $1700 \AA$ high frequency power in $\mathrm{R} 1$, between $10 \%$ and $40 \%$ of the power in quiet regions. Unfortunately, R2 is not covered by the TRACE FOV. The areas of reduced TRACE power correspond well with the power halos in MDI, although they are slightly more extended. This close correspondence of reduced chromospheric power and enhanced photospheric power has not been shown before.

The TRACE high frequency power levels are about $10 \%$ in the darkest regions (e.g. at R1) compared to the power levels of the quiet sun. The power levels around the network (e.g. at $x=$ $\left.-240^{\prime \prime}, y=0^{\prime \prime}\right)$ is about $40 \%$ of the quiet sun power. The power increase in the photosphere is only about $70 \%$. Thus, a small part of the oscillations are lost. Either they are also reflected at the $\beta \approx 1$ layer but scattered away and do not return to the surface or they are converted to other waves that we cannot detect (e.g. MHD waves that travel along the field lines upward into higher layers, which we cannot probe our current data).

The white contours connect the formation layer of the TRACE filter with the layer of $\beta \approx 1$. The outermost solid contour (for $\beta=10$ ) nicely covers the power halos in the MDI high frequency power map. The $\beta=1$ contour (dashed) surrounds the locations of decreased power in MDI where we have the highest flux density values. The power deficiency in the TRACE high frequency power map also matches the contours reasonably well, especially considering the approximations we are using in their calculation.

Our scenario can explain several observed characteristics of the power distributions. (i) Only waves with frequencies higher than the acoustic cut-off frequency $(\approx 5.5 \mathrm{mHz})$ can propagate into higher layers. Consequently, we will not find halos in the typical $p$-mode frequencies. (ii) If the $\beta \approx 1$ wave-field interaction layer is below the formation height of the TRACE UV filters, we find a decrease of power in the layers above. (iii) At least a part of the waves is reflected and depending on the angle between the wave and the magnetic field the waves can return to the photosphere and increase the oscillation power locally to form the photospheric power halos.

In the literature there are two different approaches to explain the photospheric and Ca II K power halos; (i) enhancement of acoustic emission of unknown cause, but related to moderate flux densities (Braun et al. 1992; Brown et al. 1992; Jain \& Haber 2002) and (ii) the second group does not interpret their results in terms of enhanced acoustic emission due 

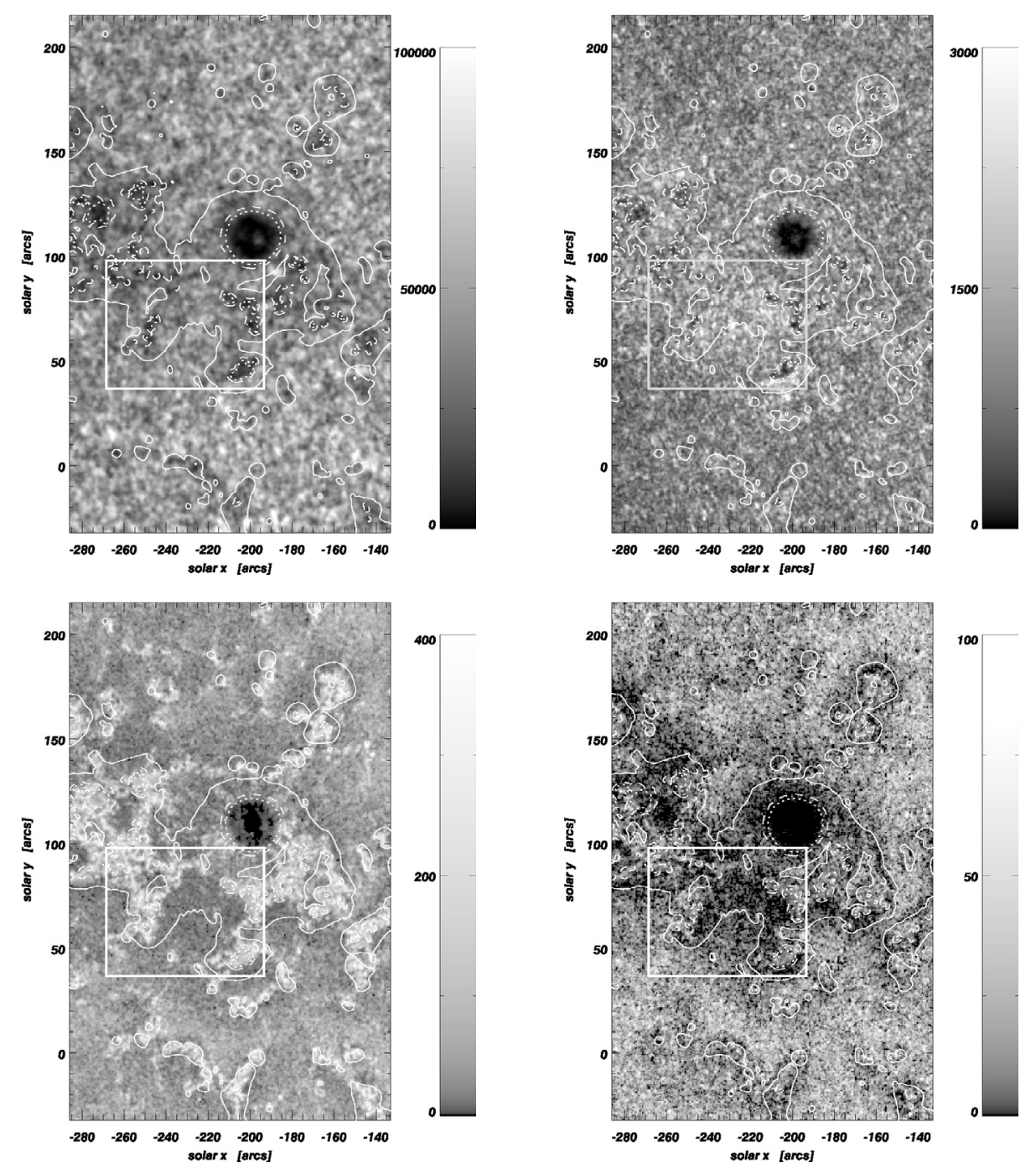

Fig. 5. Detailed comparison of the spatial distribution of MDI velocity power (top) and TRACE $1700 \AA$ intensity power (bottom). The left images show the integrated power between $2.3-4.3 \mathrm{mHz}$ ( $5 \mathrm{~min}$ range) and the right ones between $5.5-7.5 \mathrm{mHz}$ ( $2-3 \mathrm{~min}$ range). The white contours show the location of $h=500 \mathrm{~km}$ where plasma $\beta=0.5$ (dotted line), 1 (dashed line), 10 (solid line). Region R1 is outlined with the white rectangle, the numbers at the colorbars give arbitrary units.

to the lack of photospheric continuum halos. They suggest either the duction of acoustic waves by the vertical magnetic field or incompressible waves like Alfvén waves or transverse tube-waves (kink modes) (Hindman \& Brown 1998; Thomas \& Stanchfield 2000; Finsterle et al. 2004). These waves are well studied theoretically in small-scale kG fluxtubes. However, observational evidence for their existence in the solar photosphere and chromosphere from spectroscopy and polarimetry is scarce. It is clear that fluxtubes can be used to explain network and plage magnetic fields, but these fluxtubes are usually not found in the internetwork (where we find the photospheric power halos).

In the scenario we have outlined above the photospheric high frequency power halos are not sites of additional subphotospheric acoustic emission as assumed by other authors (Braun et al. 1992; Brown et al. 1992; Jain \& Haber 2002). Also one does not need to invoke MHD waves to explain the photospheric halos, as it is unclear if the internetwork atmosphere contains a magnetic field that can support such waves.
The chromospheric Ca II K halos (Braun et al. 1992; Toner \& LaBonte 1993; Thomas \& Stanchfield 2000) seem inconsistent with the above scenario but Ca II K filtergrams might well be suffering from the non-linear response of the Ca II K line to an increased photospheric driver (Carlsson \& Stein 1997). This should best be tested numerically. Simultaneous and co-spatial Ca II K, TRACE UV and photospheric Doppler measurements of a large enough FOV would be very useful to study this relationship in detail. Also, the Ca II K halos extend very far away from the AR and this area is not covered with our TRACE FOV, which might be another reason why we do not see power halos in our TRACE data. What still cannot be explained is the lack of halos in continuum intensity in the photosphere. Again, numerical modelling of the oscillation-magnetic field interaction including the calculation of the emergent spectra might shed light on this issue.

The potential field extrapolation and derived $\beta$ heights should be considered with caution for several reasons: i) the magnetic field in the photosphere is neither potential nor 
force-free, although the deviations are often less than conventionally regarded (e.g. Moon et al. 2002); ii) the VAL 3C model is not valid for strong field regions: the gas pressure in fluxtubes is usually lower and thus the plasma $\beta \approx 1$ heights as well; iii) MDI gives photospheric flux densities $|\boldsymbol{B}| \cos \phi \alpha$, with $\boldsymbol{B}$ the true magnetic field vector, $\phi$ the angle between the field vector $\boldsymbol{B}$ and the line of sight and $\alpha$ the magnetic filling factor. MDI values are thus only a lower limit for the true field $\boldsymbol{B}$ as $\cos \phi=1$ is not given in certain areas (e.g. in the sunspot penumbra) and $\alpha=1$ might be a good approximation only for a sunspot umbra. The magnetic network and plage regions have $\alpha<1$ (e.g. Stenflo 1973; Muglach \& Solanki 1992; Keller 1990). Magnetic flux tubes that make up the plage and network have $|\boldsymbol{B}| \approx 1500 \mathrm{G}$ at $h=0 \mathrm{~km}$ and $\beta<1$ even below the surface (Bellot Rubio et al. 2000).

A much more realistic approach would be the measurement of the magnetic field with spectro-polarimetry at several heights in the solar atmosphere (from $h=0 \mathrm{~km}$ in the photosphere up to $h=2000 \mathrm{~km}$, the base of the corona). Unfortunately, such measurements are not available for the oscillation data presented here. Thus we decided to use the MDI data and the potential field extrapolation as a crude approximation for the field structure.

Note that the interaction of waves and magnetic fields, especially the blocking of acoustic waves, is also of relevance for any models that require acoustic heating for the chromosphere and corona of the sun and other stars. The amount of mechanical energy channeled from below will depend on the activity cycle of the star; the stronger the activity the less energy can reach the higher layers of the stellar atmosphere.

\section{Summary}

In this paper we have presented an analysis of time sequences of MDI intensity and Doppler velocity together with filtergrams taken by TRACE at $1700 \AA$. The fact that the data was obtained co-spatially and simultaneously allows a one-to-one comparison of the spatial distribution of the oscillation power of the various observed parameters. Around the AR, high frequency halos are found in MDI velocity as well as a deficiency of high frequency power sampled at the height of the TRACE UV filter. This comparison provides phenomenological evidence for the idea that the observed modifications of wave power may be due to the interaction of the acoustic wave field with an overlying inclined magnetic field, in particular wave conversion and wave reflection.

Acknowledgements. We would like to thank the MDI and TRACE instrument teams and planners for their support during the observing campaign. SOHO is a project of international cooperation between ESA and NASA.

\section{References}

Balthasar, H., Collados, M., \& Muglach, K. 2000a, Astron. Nachr., 321,121
Balthasar, H., Collados, M., \& Muglach, K. 2000b, Proc. of the SOHO 10/GONG 2000 Workshop, Tenerife, ESA SP-464, 163

Bellot Rubio, L. R., Ruiz Cobo, B., \& Collados, M. 2000, A\&A, 535, 489

Bogdan, T. J., Hansteen, V., Carlsson, M., et al. 2003, ApJ, 599, 626

Braun, D. C., Lindsey, C., Fan, Y., \& Jefferies, S. M. 1992, ApJ, 392, 739

Brown, T. M., Bogdan, T. J., Lites, B. W., \& Thomas, J. H. 1992, ApJ, 394, L65

Bruls, J. H. M. 1993, A\&A, 269, 509

Carlsson, M., \& Stein, R. F. 1997, ApJ, 481, 500

Donea, A.-C., Lindsey, C., \& Braun, D. C. 2000, Sol. Phys., 192, 321

Finsterle, W., Jefferies, S. M., Cacciani, A., et al. 2004, Sol. Phys., 220,317

Fossum, A., \& Carlsson, M. 2004, SOHO 13 Proc., SP-457

Georgakilas, A. A., Muglach, K., \& Christopoulou, E. B. 2002, ApJ, 576,561

Gurman, J. B., \& Leibacher, J. W. 1984, ApJ, 283, 859

Handy, B., Acton, L. W., Kankelborg, C. C., et al. 1999, Sol. Phys., 187,229

Hill, F., Ladenkov, O., Ehgamberdiev, S., \& Chou, D.-Y. 2001, Helioand Asteroseismology at the Dawn of the Millennium, Proc. of the SOHO 10/GONG 2000 Workshop, ESA SP-464, 219

Hindman, B. W., \& Brown, T. M. 1998, ApJ, 504, 1029

Howard, R. F., Harvey, J. W., \& Forgach, S. 1990, Sol. Phys., 130, 295

Jain, R., \& Haber, D. 2002, A\&A, 387, 1092

Judge, P. G., Tarbell, T. D., \& Wilhelm, K. 2001, ApJ, 554, 424

Keller, C. U., Steiner, O., Stenflo, J. O., \& Solanki, S. K. 1990, A\&A, 233, 583

Krijger, J. M., Rutten, R. J., Lites, B. W., et al. 2001, A\&A, 379, 1052

Moon, Y.-J., Choe, G. S., Yun, H. S., Park, Y. D., \& Mickey, D. L. 2002, ApJ, 568, 422

Muglach, K. 2002, Il Nuovo Cimento C, 25, 647

Muglach, K. 2003, A\&A, 401, 695 (Paper I)

Muglach, K., \& O'Shea, E. 2001, Proc. of the INTAS Workshop on MHD waves in Astrophysical Plasmas, ed. J. L. Ballester, \& B. Roberts, 155

Muglach, K., \& Solanki, S. K. 1992, A\&A, 263, 301

O'Shea, E., Muglach, K., \& Fleck, B. 2002, A\&A, 387, 642

O'Shea, E., Muglach, K., \& Fleck, B. 2003, 12th Workshop on Cool Stars, Stellar Systems and the Sun, 601

Rosenthal, C. S., Bogdan, T. J., Carlsson, M., et al. 2002, ApJ, 564, 508

Scherrer, P. H., Bogart, R. S., Bush, R. I., et al. 1995, Sol. Phys., 162, 129

Seehafer, N. 1978, Sol. Phys., 58, 215

Settele, A., Zhugzhda, Y. D., \& Staude, J. 1999, Astron. Nachr., 320, 147

Settele, A., Staude, J., \& Zhugzhda, Y. D. 2001, Sol. Phys., 202, 281

Settele, A., Carroll, T. A., Nickelt, I., \& Norton, A. A. 2002a, A\&A, 386,1123

Settele, A., Sigwarth, M., \& Muglach, K. 2002b, A\&A, 392, 1095

Stenflo, J. O. 1973, Sol. Phys., 32, 41

Thomas, J. H., \& Stanchfield, D. C. H. 2000, ApJ, 537, 1086

Toner, C. G., \& LaBonte, B. J. 1993, ApJ, 415, 847

Uitenbroek, H. 2004, SOHO 13 Proc., SP-547

Vernazza, J. E., Avrett, E. H., \& Loeser, R. 1981, ApJS, 45, 635

Zhugzhda, Iu. D., \& Dzhalilov, N. S. 1984a, A\&A, 132, 45

Zhugzhda, Iu. D., \& Dzhalilov, N. S. 1984b, A\&A, 132, 52

Žugžda, Y. D., Locāns, V., \& Staude, J. 1983, Sol. Phys., 82, 369

Žugžda, Y. D., Locāns, V., \& Staude, J. 1987, Astron. Nachr., 308, 257 